\title{
In situ mask designed for selective growth of InAs quantum dots in narrow regions developed for molecular beam epitaxy system
}

\author{
Shunsuke Ohkouchi ${ }^{\mathrm{a}}$ \\ The Femtosecond Technology Research Association (FESTA), 5-5 Tokodai, Tsukuba, Ibaraki 300-2635, Japan \\ Yusui Nakamura \\ The Femtosecond Technology Research Association (FESTA), 5-5 Tokodai, Tsukuba, Ibaraki 300-2635, Japan \\ and Graduate School of Science and Technology, Kumamoto University, 2-39-1 Kurokami, Kumamoto \\ 860-8555, Japan \\ Naoki lkeda \\ The Femtosecond Technology Research Association (FESTA), 5-5 Tokodai, Tsukuba, Ibaraki 300-2635, Japan \\ and National Institute of Advanced Industrial Science and Technology (AIST), 1-1-1 Umezono, Tsukuba, \\ Ibaraki 305-8568, Japan \\ Yoshimasa Sugimoto and Kiyoshi Asakawa \\ The Femtosecond Technology Research Association (FESTA), 5-5 Tokodai, Tsukuba, Ibaraki 300-2635, Japan \\ and Center for TARA, University of Tsukuba, 1-1-1 Tennoudai, Tsukuba, Ibaraki 305-8577, Japan
}

(Received 6 April 2007; accepted 17 June 2007; published online 27 July 2007)

\begin{abstract}
We have developed an in situ mask that enables the selective formation of molecular beam epitaxially grown layers in narrow regions. This mask can be fitted to a sample holder and removed in an ultrahigh-vacuum environment; thus, device structures can be fabricated without exposing the sample surfaces to air. Moreover, this mask enables the observation of reflection high-energy electron diffraction during growth with the mask positioned on the sample holder and provides for the formation of marker layers for ensuring alignment in the processes following the selective growth. To explore the effectiveness of the proposed in situ mask, we used it to grow quantum dot (QD) structures in narrow regions and verified the perfect selectivity of the QD growth. The grown QDs exhibited high optical quality with a photoluminescence peak at approximately $1.30 \mu \mathrm{m}$ and a linewidth of $30 \mathrm{meV}$ at room temperature. The proposed technique can be applied for the integration of microstructures into optoelectronic functional devices. (c) 2007 American Institute of Physics. [DOI: 10.1063/1.2756624]
\end{abstract}

\section{INTRODUCTION}

Selective area epitaxy (SAE) has attracted attention because of its tremendous potential for achieving the monolithic integration of optoelectronic devices and lowdimensional semiconductor structures, including laser diodes, optical switches, and quantum dots. ${ }^{1-3}$ Various results have been obtained through the implementation of this technique, including molecular beam epitaxy (MBE), metal organic vapor phase epitaxy (MOVPE), and chemical beam epitaxy (CBE) growth. ${ }^{4-6}$ SAE enhances the effectiveness of crystal growth techniques by defining local areas in which materials can be grown using oxide masks, self-aligned substrate masks, or mechanical shadow masks. ${ }^{7-11}$ Among these methods, the shadow-mask-based SAE method has various advantages over the oxide- and substrate-mask-based ones due to its simplicity and suitability for multiple SAE steps. For example, in the former, no polycrystalline coatings need to be removed, nor does it involve complicated substrate surface treatments. Mechanical masks for selective growth

\footnotetext{
${ }^{a)}$ Present address: Fundamental and Environmental Research Laboratories, NEC Corp., 34 Miyukigaoka, Tsukuba, Ibaraki 305-8501, Japan; Electronic mail: s-ohkouchi@ab.jp.nec.com
}

were first used by Cho and Reinhart for the in situ fabrication of waveguide structures in integrated optics in $1972 .{ }^{10}$ Furthermore, the selective growth in preferentially etched channels on a GaAs substrate was demonstrated by Tsang and

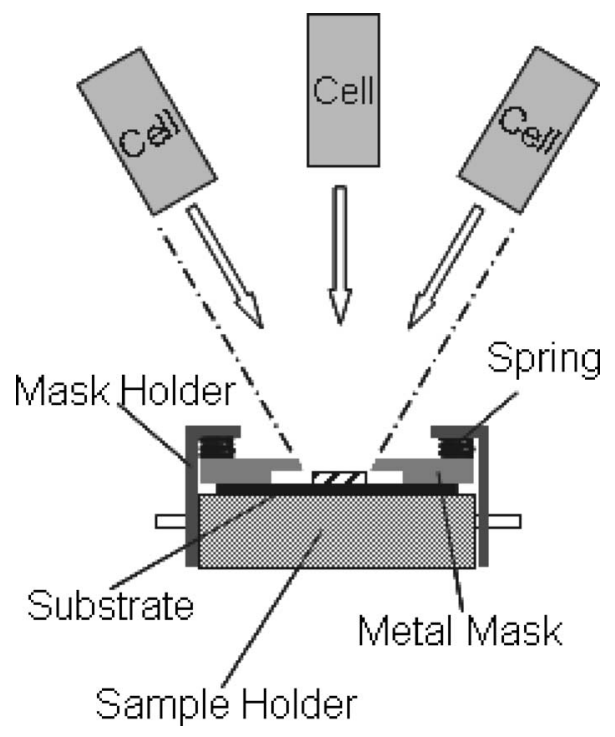

FIG. 1. Schematic diagram of the procedures of epitaxial growth using the in situ mask. 
Ilegems in $1977 .{ }^{11}$ In their study, silicon masks with etched windows were used because of their mechanical strength and their reusability in MBE growth since the polycrystalline GaAs epilayer can be easily removed after growth. In addition, the excellent selective formation of both $\mathrm{CdTe}$ and $\mathrm{Au}$ layers by employing a multiple mask technique was recently reported. ${ }^{12}$

In this article, we report on an in situ mask that enables the selective formation of a molecular beam epitaxially grown layer in a narrow region. The mask was designed from the viewpoint of facilitating to integrating lowdimensional semiconductor structures such as quantum dots (QDs) into optoelectronic devices. The effectiveness of the mask was demonstrated by the selective formation of QD structures in narrow regions.

\section{IN SITU MASK}

\section{A. General principle}

In situ device processing is an attractive approach to simplify complex device-fabrication technologies. Ideally, it is preferable that the entire device structure can be fabricated within a vacuum system without an intermediate exposure to air. This will result in a significant simplification of the fabrication process and a subsequent increase in device yields. Figure 1 shows a schematic diagram illustrating the principles of epitaxial growth using the proposed in situ mask. A mechanical mask with window-type openings is held between the evaporating sources and substrate during MBE growth. We designed this special mask such that it could be fitted to the sample holder used for MBE. Made of molybdenum, this mask can be fitted and removed in an ultrahighvacuum (UHV) environment. The molecular beams of constituent materials such as In, Ga, and As atoms can be irradiated onto the sample surface through the window openings in the mask. The rectangular windows are fabricated by mechanical machining and chemical polishing. Several windows are formed in the mask with typical dimensions of $300 \mu \mathrm{m} \times 2 \mathrm{~mm}$. The edges of the windows are beveled to an angle of $40^{\circ}$ to prevent shadowing from the incident molecular beams during growth. The dimensions of the in situ mask are nearly identical to those of the sample holder. The diameter of the sample holder is approximately $40 \mathrm{~mm}$. The metal mask is mounted in the mask holder by using three springs that keep the mask firmly pressed against the substrate surface around the fringe. The reverse side of the center region of the metal mask, where selective growth is performed, is ground to a depth of $100 \mu \mathrm{m}$ to prevent the contact between the metal mask and substrate surface. Setting the mask on the sample holder during growth permits selective growth only in the open regions.

\section{B. Additional functions}

The proposed in situ mask has two additional functions. The first function is that it allows for the observation of reflection high-energy electron diffraction (RHEED) during growth. In the shadow-mask-based methods, typically, it is not possible to observe RHEED during growth since the mask shadows the RHEED-incident electron beams. To solve

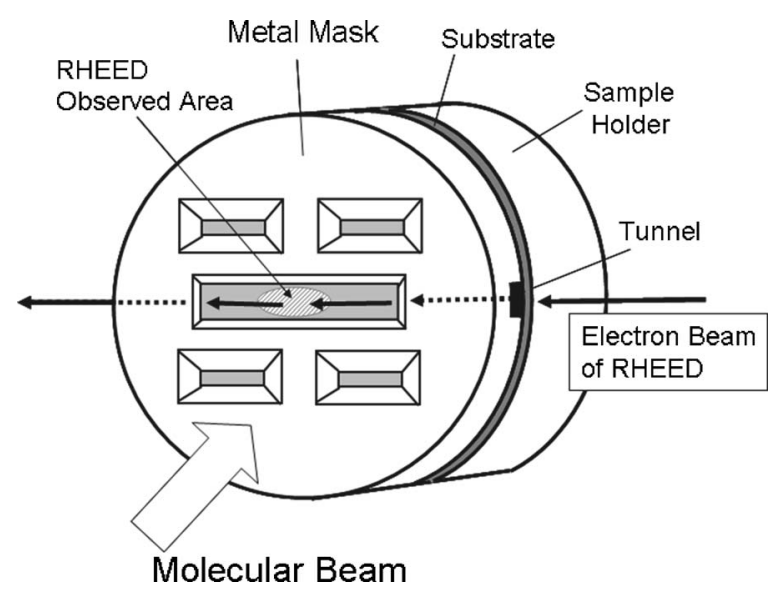

FIG. 2. Schematic diagram of the principle of the RHEED observation using the proposed mask. The tunnel was formed on the reverse side of the metal mask. The RHEED electron beam enters through the tunnel entrance with a small incident angle and the diffracted beam at the observed area exits through the tunnel exit.

this problem, we fabricated a tunnel for the electron beam on the reverse side of the metal mask, as shown in Fig. 2. Additionally, another window was fabricated in the center region, where the electron beam for RHEED is diffracted, and the molecular beams were irradiated under the same conditions. The RHEED electron beam enters the tunnel at a small incident angle of less than $0.5^{\circ}$, and the diffracted beam exits from the tunnel. This configuration for enabling RHEED observation can be achieved by precisely adjusting the position and rotation of the sample holder relative to the incident electron beam. By using the proposed mask, it is possible to observe RHEED with the mask positioned on the sample holder.

The second additional function is the fabrication of marker layers to ensure alignment in the processes following the selective growth. If the selectively grown layer is very thin, it cannot be viewed using an optical microscope and/or a scanning electron microscope. In these cases, additional marker layers can be grown using this function. For this purpose, we fabricated another small window and a shutter mechanism on the mask holder, as shown in Fig. 3. The additional windows are fabricated outside the selectively grown regions. The shutter can be opened and closed by

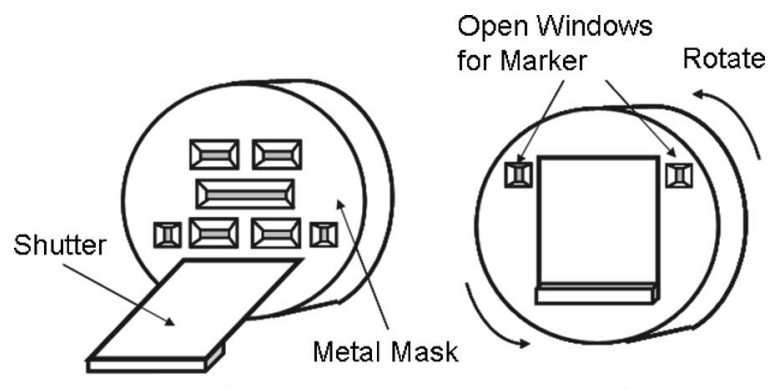

(a)

(b)

FIG. 3. Schematic diagram of the principle of fabricating a marker layer using the proposed mask. During the selective growth, the shutter is opened, as shown in (a). After the selective growth, the sample is rotated by $180^{\circ}$, as shown in (b), such that the shutter is closed and the marker layers are grown only in the additional small open areas. 


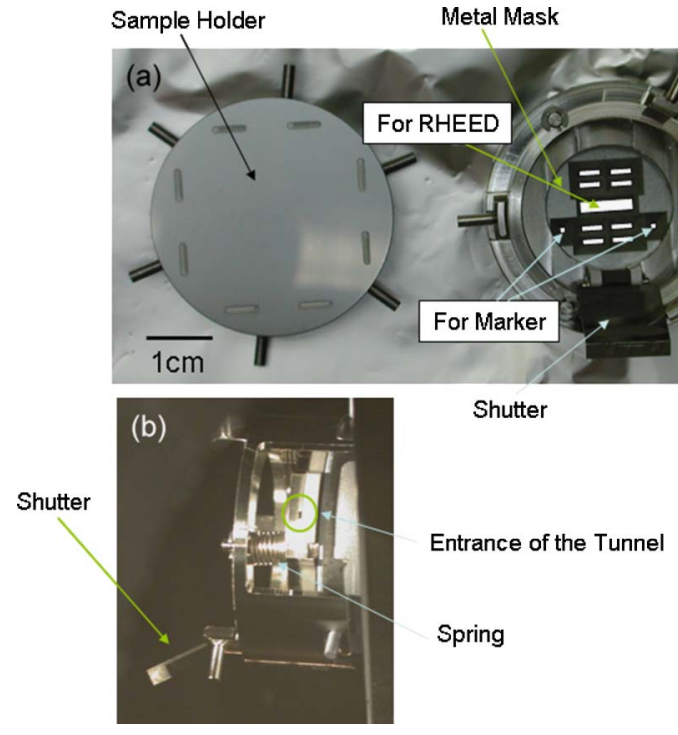

FIG. 4. (Color online) Photographs of a sample holder and the in situ mask. (a) shows the front views of the sample holder and in situ mask, and (b) shows a side view of the in situ mask mounted onto the sample holder. The size of the mask is approximately $40 \mathrm{~mm}$ (1.5 in.) in diameter.

rotating the sample holder due to the weight of the shutter itself. To ensure this shutter movement, a weight is attached to the end of the shutter. During a selective growth, the shutter is opened, as shown in Fig. 3(a). After a selective growth, the temperature of the sample is lowered to prevent the desorption of V-group element atoms on the sample surface due to the interruption of the irradiation of flux by the shutter. The sample holder is rotated by $180^{\circ}$ using the mechanism of a MBE manipulator, as shown in Fig. 3(b), such that the shutter is closed and the marker layers can be grown only in the additional small open areas. The material chosen for fabricating the marker layer generally depends on the growth systems. In this study, a GaAs layer grown at a low temperature was used as a marker layer.

\section{Fabricated mask}

Figure 4(a) shows the photographs of a special mask with the sample holder developed in this study. The sample holder has six pins on its side. The three pins on the lower level are used for fixing the sample holder to the MBE manipulator, and the remaining three pins on the upper level are used for fitting the mask holder to the sample holder; the mask holder features slots corresponding to the upper level pins. The mask holder also has three pins on its side; these pins allow for the placement of the mask holder in a cart for transport in transfer modules or for transfer to the MBE growth system using a transfer rod. The metal mask is mounted and fixed to the mask holder equipped with a shutter. It has an array of open square windows for selective growth, a large window in the center region for RHEED observation, and two small windows for the fabrication of the marker layers. On the sample holder, shallow grooves are formed to allow for the correct determination of the relative position of the substrate and metal mask by mounting the substrate along the grooves. Figure 4(b) shows a photograph of the side view of the mask holder attached to the sample

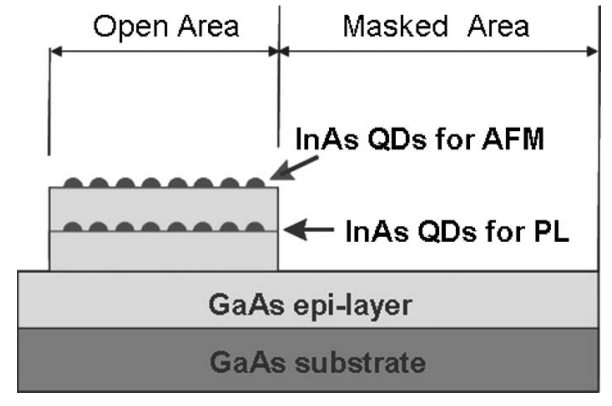

FIG. 5. Schematic diagram of a sample structure. The selectively grown layers were formed using the in situ mask.

holder, which is mounted on the MBE manipulator for selective growth. The entrance of the electron beam for RHEED observation is located on one side of the mask holder. The dimensions of the entrance are $1 \times 2 \mathrm{~mm}^{2}$.

\section{SELECTIVE GROWTH USING IN SITU MASK}

\section{A. Growth procedures}

To explore the effectiveness of the proposed in situ mask, we used it to selectively grow QD structures in narrow regions. All the fabrication processes were carried out in an UHV environment without exposing the sample surface to air. Prior to the growth process, the in situ mask was baked at $600{ }^{\circ} \mathrm{C}$ for $2 \mathrm{~h}$ in the UHV chamber, which was connected to the MBE chamber through the UHV tunnel, and it was kept in the UHV environment until the selective growth using the mask was achieved. These processes protected the sample surface from contamination during the growth. This was also confirmed by another experimental result; the photoluminescence (PL) spectrum of the QDs in the masked region revealed no degradation even after the process of mounting and removing the mask during the growth sequence. ${ }^{13}$ The cross-sectional structure of the sample is shown in Fig. 5. First, we grew a 100-nm-thick GaAs buffer layer at $560{ }^{\circ} \mathrm{C}$ without the in situ mask. Then, after setting the in situ mask on the sample, we grew GaAs $(20 \mathrm{~nm}) / \operatorname{AlGaAs}(20 \mathrm{~nm}) / \mathrm{GaAs}(100 \mathrm{~nm})$ layers. An example of a RHEED pattern observed during this growth is shown in Fig. 6. This image was produced by the diffracted electron beam that penetrated through the tunnel when the in situ mask was attached. The growth temperature of the QDs was determined from the transition temperature, which was

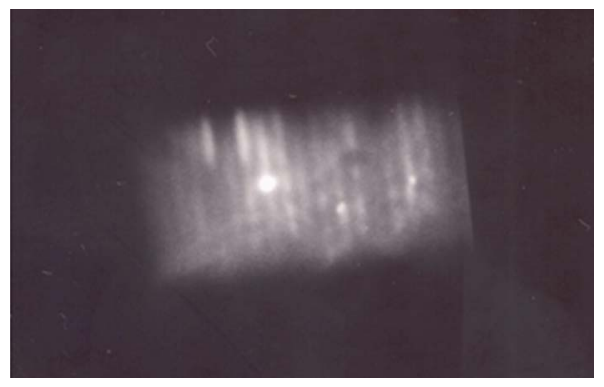

FIG. 6. RHEED pattern of the open regions during the growth of the GaAs layer after mounting the mask onto the sample holder. The incident electron beam is along the $[\overline{1} 10]$ azimuth. 


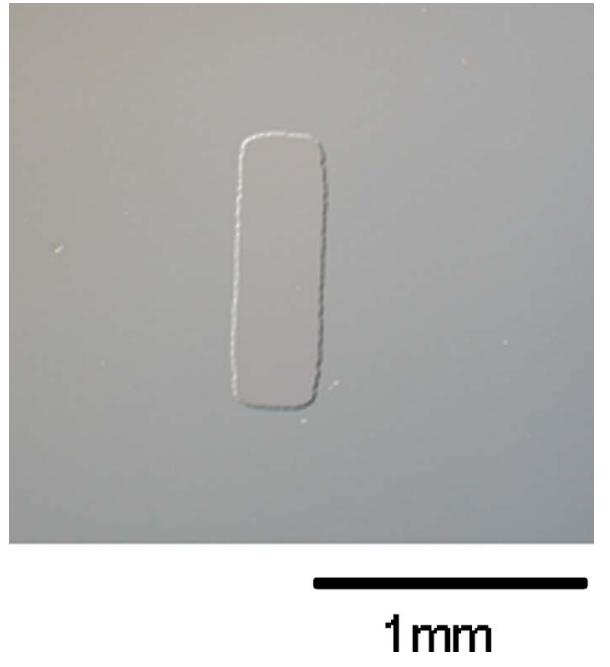

FIG. 7. (Color online) Optical microscope image of the GaAs marker layer grown by the molecular beam supply through the additional windows.

determined by the change in the surface reconstruction of the RHEED pattern.

Then, a 2.6 ML (monolayer) InAs QD layer was grown at $480{ }^{\circ} \mathrm{C}$ by the two-step growth method, which provides both satisfactory uniformity and high density for optical measurement. ${ }^{14}$ This method comprises two stages: In the first stage, InAs is grown rapidly until its thickness just exceeds the critical thickness required to form high-density nuclei as a template. In the second stage, InAs is grown continuously at a lower rate to enlarge and homogenize the QDs. In actual growth, after rapid $\left(0.2 \mathrm{ML} \mathrm{s}^{-1}\right)$ deposition of a 2.0 ML InAs layer, the rate was reduced to $0.2 \mathrm{MLs}^{-1}$ by shutter control, and the remaining 0.6 ML was deposited at a lower rate. After the growth of a QD layer, a capped $\mathrm{In}_{0.2} \mathrm{Ga}_{0.8} \mathrm{As}$ layer $\left(4 \mathrm{~nm}\right.$ ) was grown at $430{ }^{\circ} \mathrm{C}$ to shift the PL of the QDs up to $1.3 \mu \mathrm{m}$; this procedure was employed with a view to applying it in the fabrication of long-wavelength optical communication devices. ${ }^{15}$ In addition, GaAs $(96 \mathrm{~nm}) / \operatorname{AlGaAs}(20 \mathrm{~nm}) / \mathrm{GaAs}(20 \mathrm{~nm})$ layers followed. Finally, one more InAs QD layer was grown at $480^{\circ} \mathrm{C}$ by using the two-step-growth method once again; this layer was used to measure the QD density by atomic force microscopy (AFM).

Figure 7 shows an optical microscope image of a marker layer. This layer was composed of GaAs grown at a low temperature and was formed outside the selectively grown region. During the selective growth, the shutter was opened, as shown in Fig. 3(a). After the selective growth, the temperature of the sample was lowered to $200{ }^{\circ} \mathrm{C}$. Then, the sample holder was rotated by $180^{\circ}$ using a MBE manipulator, as shown in Fig. 3(b). Consequently, the shutter was closed and the selectively grown layers were covered. Under this configuration, the marker layers could only be grown in the additional small open areas. The dimensions of the marker layers were $300 \mu \mathrm{m} \times 1 \mathrm{~mm}$. Although these marker layers were not used for the subsequent fabrication process in this experiment, it is a powerful function for ensuring the alignment in the subsequent processes, especially in the case wherein the selectively grown layer cannot be recognized by

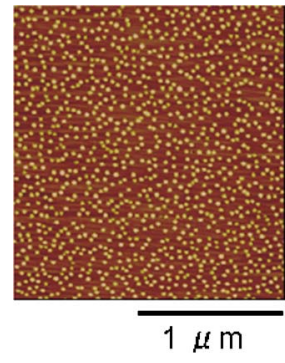

(a)

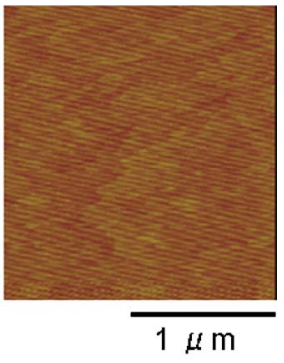

(b)
FIG. 8. (Color online) AFM images of (a) an open region and (b) a masked region. High-density QDs are formed in the open region, whereas no QDs are observed in the masked region.

other methods such as by using an optical microscope.

\section{B. Characteristics}

First, we observed the AFM image of a top InAs QD layer grown selectively in the open regions of the proposed in situ mask. Figure 8(a) shows the AFM image in which the grown QDs exhibit a high density of $3 \times 10^{10} \mathrm{~cm}^{-2}$. In contrast, the AFM image of the regions covered by the in situ mask shown in Fig. 8(b) reveals no QDs.

Next, we measured the optical characteristics of the sample. Figure 9 shows the PL spectra at room temperature. In the open region, a distinct peak is seen at $1.30 \mu \mathrm{m}$, where the linewidth is as narrow as $30 \mathrm{meV}$. This PL spectrum was measured in the center region of the selectively grown area. In contrast, in other regions covered by the in situ mask, no PL peak was observed. These results show that the proposed method using the in situ mask allows for the perfect selective growth of high-quality QDs.

The intensity of the PL in the open regions is almost identical in all the grown areas, except for the regions within approximately $50 \mu \mathrm{m}$ from the edges. That is, there are no QDs near the edge of the open areas. This shrinkage of the selectively grown areas is probably due to the thermal evaporation of the QDs by heat radiation from the mask, which was positioned close to the sample surface. QDs are supposed to be sensitive to heat radiation as compared with other thin films and are easily destroyed. This reduction in

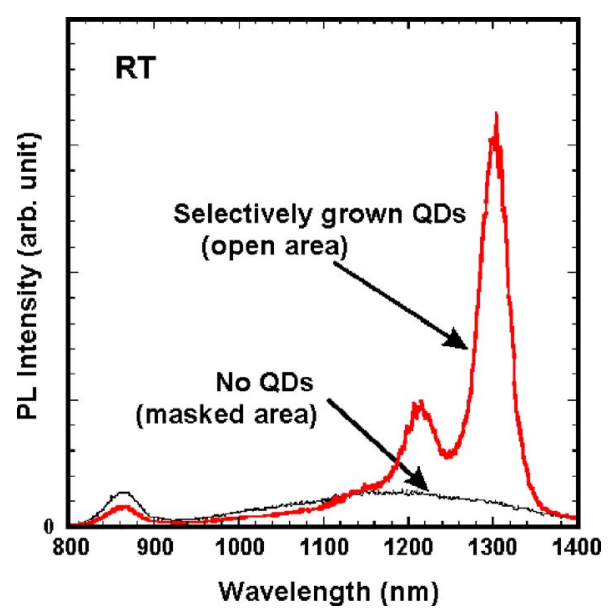

FIG. 9. (Color online) Room temperature photoluminescence spectra of the InAs dots formed in masked and open areas. 


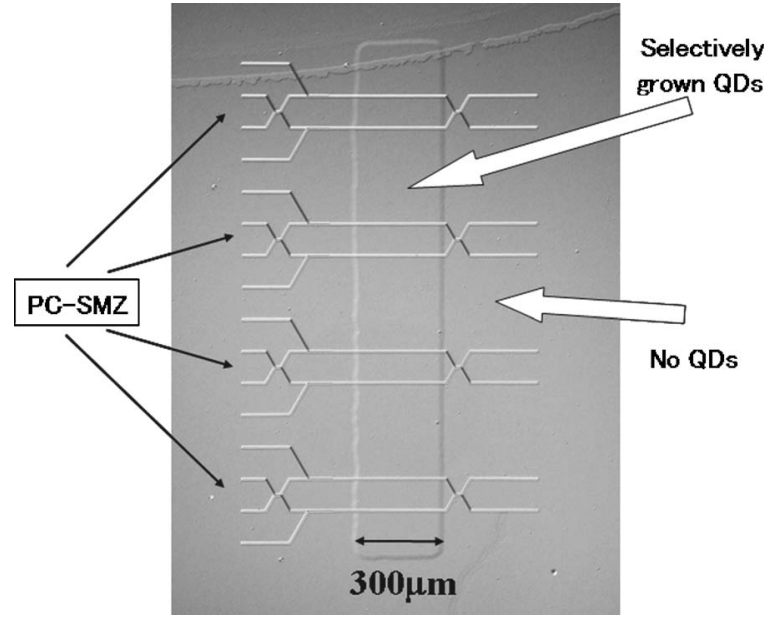

FIG. 10. Optical microscope image of PC-SMZ devices which were fabricated on a selectively grown QD layer using the in situ mask.

the selectively grown regions should be taken into account when designing the device structures using this technique.

\section{Applications}

This method can be applied to the integration of QD structures into optoelectronics devices. One specific application wherein this method can be employed is the fabrication of our proposed photonic-crystal-based symmetrical MachZehnder-type (PC-SMZ) all-optical switch. ${ }^{16,17}$ In this device, QDs are used as optical nonlinear materials. This device requires a large number of high-uniformity, high-quality QDs in the PC waveguide region. To achieve the switching of signal pulses, the open area should be defined as the phase-shift region of the waveguide. Figure 10 shows an optical microscope photograph of a sample of PC-SMZ device fabricated on a selectively grown QD layer. Thus, we have successfully realized a PC-SMZ structure with an optical nonlinear waveguide. Achieving the selective growth of QDs is one of the key issues in designing optoelectronic functional devices. The significance of the proposed method is evident because it can be used to fabricate not only our proposed PC-SMZ device but also future high-performance functional devices, such as multiwavelength light-emitting sources and photonic crystal microcavities. ${ }^{18,19}$ The proposed method using in situ mask provides additional latitude in designing optoelectronic and electronic devices.

\section{ACKNOWLEDGMENT}

This work was supported by the New Energy and Industrial Technology Development Organization (NEDO) within the framework of the Femtosecond Technology Project.

${ }^{1}$ Y. Furushima, H. Yamazaki, K. Kudo, Y. Sakata, Y. Okunuki, Y. Sasaki, and T. Sasaki, Jpn. J. Appl. Phys., Part 1 38, 1234 (1999).

${ }^{2}$ S. Nakamura et al., IEEE Photonics Technol. Lett. 12, 425 (2000).

${ }^{3}$ Y. Nakamura, N. Ikeda, S. Ohkouchi, Y. Sugimoto, H. Nakamura, and K. Asakawa, Physica E (Amsterdam) 21, 551 (2004).

${ }^{4}$ W. T. Tsang and A. Y. Cho, Appl. Phys. Lett. 32, 491 (1978).

${ }^{5}$ P. Demester, L. Buydens, I. Moerman, D. Lootens, and P. Van Daele, J. Cryst. Growth 107, 161 (1991).

${ }^{6}$ N. Y. Li, H. K. Dong, Y. M. Hsin, T. Nakamura, P. M. Asbeck, and C. W. Tu, J. Vac. Sci. Technol. B 13, 664 (1995).

${ }^{7}$ C. Ghosh and R. L. Layman, Appl. Phys. Lett. 45, 1229 (1984).

${ }^{8}$ U. Hilburger et al., J. Cryst. Growth 201/202, 574 (1999).

${ }^{9}$ S. Malzer, M. Kneissl, P. Kiesel, K. H. Gulden, X. X. Wu, J. S. Smith, and G. H. Döhler, J. Vac. Sci. Technol. B 14, 2175 (1996).

${ }^{10}$ A. Y. Cho and F. K. Reinhart, Appl. Phys. Lett. 21, 355 (1972).

${ }^{11}$ W. T. Tsang and M. Ilegems, Appl. Phys. Lett. 31, 301 (1977).

${ }^{12}$ Y. Y. Luo, A. Cavus, and M. C. Tamargo, J. Electron. Mater. 26, 511 (1997).

${ }^{13}$ S. Ohkouchi, Y. Nakamura, H. Nakamura, and K. Asakawa, Jpn. J. Appl. Phys., Part 1 44, 5677 (2005).

${ }^{14}$ Y. Nakamura, H. Nakamura, S. Ohkouchi, N. Ikeda, Y. Sugimoto, and K. Asakawa, IOP Pub. Ltd., Inst. Phys. Conf. Ser. No. 174, 2003, p. 133.

${ }^{15}$ Y. Nakata, K. Mukai, M. Sugawara, K. Ohtsubo, H. Ishikawa, and N. Yokoyama, J. Cryst. Growth 208, 93 (2000).

${ }^{16}$ Y. Sugimoto, N. Ikeda, N. Carlsson, K. Asakawa, N. Kawai, and K. Inoue, J. Appl. Phys. 91, 922 (2002).

${ }^{17}$ H. Nakamura et al., Opt. Express 12, 6606 (2004).

${ }^{18}$ A. Imamoglu, D. D. Awschalom, G. Burkard, D. P. DiVincenzo, D. Loss, M. Sherwin, and A. Small, Phys. Rev. Lett. 83, 4204 (1999).

${ }^{19}$ K. Hennessy et al., Appl. Phys. Lett. 83, 3650 (2003). 